\title{
Patient-prosthesis mismatch after minimally invasive aortic valve replacement
}

\author{
Grzegorz Filip ${ }^{1 *}$, Radoslaw Litwinowicz ${ }^{1 *}$, Boguslaw Kapelak ${ }^{1}$, Magdalena Bryndza ${ }^{1}$, Magdalena Bartus², \\ Janusz Konstanty-Kalandyk ${ }^{1}$, Piotr Ceranowicz ${ }^{3}$, Maciej Brzezinski ${ }^{4}$, Sameer Gafoor ${ }^{5}$, Krzysztof Bartus ${ }^{1}$ \\ 1Department of Cardiovascular Surgery and Transplantology, Jagiellonian University and John Paul II Hospital, Krakow, Poland \\ 2Department of Pharmacology, Jagiellonian University, Krakow, Poland \\ 3Department of Physiology, Jagiellonian University, Krakow, Poland \\ ${ }^{4}$ Department of Cardiac and Vascular Surgery, Medical University of Gdansk, Gdansk, Poland \\ ${ }^{5}$ CardioVascular Centre Frankfurt, Germany and Swedish Medical Centre Seattle, United States \\ *Grzegorz Filip and Radoslaw Litwinowicz are first authors of this manuscript and have contributed equally to the content of this paper.
}

\section{INTRODUCTION}

Compared to conventional aortic valve replacement (con-AVR, sternotomy), minimally invasive aortic valve replacement (mini-AVR) is associated with lower morbidity and mortality in high-risk patients. However, mini-AVR is technically challenging and more demanding. Limited access to the heart and a smaller operative field may lead to incorrect prosthesis size selection with greater risk of postoperative patient-prosthesis mismatch (PPM).

Patient-prosthesis mismatch occurs when the indexed effective orifice area (EOAi) of the implanted valve prosthesis is too small compared to the patient's body surface area (BSA). PPM is a strong and independent predictor of cardiac events, left ventricular overload, and mortality [1, 2]. To date, the literature on PPM after mini-AVR is very limited.

The aim of our study was to analyse the prevalence of PPM after mini-AVR and the effect of PPM on post-operative transvalvular gradient.

\section{METHODS}

A total of 150 patients were enrolled in the study; 74 patients who underwent mini-AVR and 76 patients who underwent con-AVR. In each group all procedures were performed by a group of four experienced surgeons who decided which method to use as well as which prosthesis type and size. Patients were similar in terms of age, body mass index, sex, and comorbidities.

The EOAi was calculated by dividing the corresponding EOA of each valve type and size (registered in vitro values published by each manufacturer) by each patient's BSA.
BSA was calculated using methods described in a similar study [3]. PPM was defined as not clinically significant if the projected EOAi was $>0.85$, moderate if it was $>0.65$ and $\leq 0.85$, and severe if it was $\leq 0.65 \mathrm{~cm}^{2} / \mathrm{m}^{2}[4]$.

\section{RESULTS \\ Patients-prosthesis mismatch}

Mean EOAi of all AVR patients was $0.97 \mathrm{~cm}^{2} / \mathrm{m}^{2}$, with PPM present in $31 \%(\mathrm{n}=46)$. Mean EOAi in the PPM group was $0.73 \pm 0.8 \mathrm{~cm}^{2} / \mathrm{m}^{2}$ versus $1.07 \pm 0.15 \mathrm{~cm}^{2} / \mathrm{m}^{2}$ in the group without PPM $(p<0.001)$. Severe PPM (EOAi $\left.\leq 0.65 \mathrm{~cm}^{2} / \mathrm{m}^{2}\right)$ was present in $3.3 \%(\mathrm{n}=5)$, and moderate PPM $\left(0.65 \mathrm{~cm}^{2} / \mathrm{m}^{2}<\mathrm{EOAi} \leq 0.85 \mathrm{~cm}^{2} / \mathrm{m}^{2}\right)$ was present in $27.3 \%$ of all AVR patients.

In mini-AVR, the incidence of PPM was $39 \%$, with mean EOAi $0.91 \pm 0.16 \mathrm{~cm}^{2} / \mathrm{m}^{2}$ and was higher compared to con-AVR, where the incidence of PPM was $22 \%$, with mean EOAi $1.02 \pm 0.23 \mathrm{~cm}^{2} / \mathrm{m}^{2}(p=0.04$ for PPM and $p=0.003$ for EOAi).

\section{Postoperative transprosthetic gradient}

In all AVR patients, there was a significantly higher peak/mean postoperative transprosthetic gradient in the PPM group in comparison to the group without PPM $(27.9 / 16.6 \mathrm{mmHg}$ vs. $23.5 / 13.6 \mathrm{mmHg}, \mathrm{p}<0.007$ and $\mathrm{p}<0.001)$. This was not the case in mini-AVR, where there was no difference in peak/mean postoperative transprosthetic gradient between the two groups $(26.7 / 15.6 \mathrm{mmHg}$ vs. $26.1 / 14.7 \mathrm{mmHg}$, $\mathrm{p}>0.05)$. However, there was a significantly higher postoperative peak/mean transprosthesis gradient in con-AVR patients

Address for correspondence:

Radoslaw Litwinowicz, MD, PhD, Department of Cardiovascular Surgery and Transplantology, Jagiellonian University, and John Paul II Hospital, ul. Prądnicka 80, 31-202 Kraków, Poland, tel: +48 1261430 75, fax: +48 1261425 25, e-mail: radek.litwinowicz@gmail.com

Received: 20.11.2017 Accepted: 07.02.2018

Kardiologia Polska Copyright (c) Polish Cardiac Society 2018 
Table 1. Relationship between the incidence of patient-prosthesis mismatch (PPM), mean effective orifice area index (EOAi), peak aortic valve gradient, and mean aortic valve and the prosthesis size

\begin{tabular}{|c|c|c|c|c|c|}
\hline \multirow[t]{2}{*}{ Aortic prosthesis size } & \multicolumn{2}{|c|}{ Mini-AVR $(n=74)$} & \multicolumn{2}{|c|}{ Con-AVR $(n=76)$} & \multirow[t]{2}{*}{$\mathbf{p}$} \\
\hline & No-PPM & PPM & No-PPM & PPM & \\
\hline Small size [19 mm]: & $33 \%(n=1)$ & $67 \%(n=2)$ & $40 \%(n=2)$ & $60 \%(n=3)$ & $>0.05$ \\
\hline EOAi $\left[\mathrm{cm}^{2} / \mathrm{m}^{2}\right]$ & 0.99 & $0.75 \pm 9.65$ & 0.95 & $0.81 \pm 0.01$ & $>0.05$ \\
\hline Peak AVG [mmHg] & 74 & $27 \pm 0.71$ & $36 \pm 1.41$ & $36 \pm 11.53$ & $>0.05$ \\
\hline Mean AVG [mmHg] & 38 & $17 \pm 1.41$ & $18 \pm 2.82$ & $21 \pm 5.29$ & $>0.05$ \\
\hline Medium size [21 and $23 \mathrm{~mm}$ ]: & $58 \%(n=37)$ & $42 \%(n=27)$ & $79 \%(n=46)$ & $21 \%(n=12)$ & $>0.05$ \\
\hline EOAi $\left[\mathrm{cm}^{2} / \mathrm{m}^{2}\right]$ & $1.01 \pm 0.09$ & $0.74 \pm 0.09$ & $1.09 \pm 0.19$ & $0.7 \pm 0.7$ & 0.007 \\
\hline Peak AVG $[\mathrm{mmHg}]$ & $25.61 \pm 14.18$ & $26.6 \pm 13.84$ & $21.6 \pm 8.95$ & $29.56 \pm 8.99$ & $>0.05$ \\
\hline Mean AVG [mmHg] & $15.47 \pm 8.04$ & $14.61 \pm 4.67$ & $12.6 \pm 5.87$ & $18.11 \pm 6.17$ & $>0.05$ \\
\hline Large size [27 mm]: & $100 \%(n=7)$ & $0 \%(n=0)$ & $85 \%(n=13)$ & $15 \%(n=2)$ & $>0.05$ \\
\hline EOAi $\left[\mathrm{cm}^{2} / \mathrm{m}^{2}\right]$ & $1.08 \pm 0.11$ & - & $1.18 \pm 0.13$ & $0.77 \pm 0.03$ & $>0.05$ \\
\hline Peak AVG [mmHg] & $20.5 \pm 3.27$ & - & $18.01 \pm 5.86$ & 21 & $>0.05$ \\
\hline Mean AVG [mmHg] & $11.71 \pm 3.46$ & - & $11.74 \pm 5$ & 15 & $>0.05$ \\
\hline
\end{tabular}

Data are shown as mean \pm standard deviation. AVG — aortic valve gradient; AVR — aortic valve replacement; con — conventional; mini — minimally invasive

with PPM compared to those without PPM (30.4/18.5 mmHg vs. $21 / 12.7 \mathrm{mmHg}$, $\mathrm{p}<0.002$ and $\mathrm{p}<0.001)$.

\section{Aortic prosthesis size}

In medium aortic prosthesis size a significantly lower mean EOAi was found in patients with mini-AVR in comparison to con-AVR patients $\left(0.9 \pm 0.16 \mathrm{~cm}^{2} / \mathrm{m}^{2}\right.$ vs. $1.01 \pm 0.24 \mathrm{~cm}^{2} / \mathrm{m}^{2}$, $p=0.007)$. There was no significant differences in mean EOAi, peak aortic valve gradient, or mean aortic valve gradient in other prosthesis sizes (Table 1).

\section{DISCUSSION}

Globally, the incidence of PPM after median sternotomy is estimated at between $20 \%$ and $70 \%$ [1]. Our study results are similar: overall moderate PPM was observed in about $27.3 \%$ of patients, and $3.3 \%$ of patients had severe PPM [5, 6]. In contrast to a previous study [5], we found that the incidence of PPM was higher in mini-AVR compared to con-AVR. This may be due to several reasons (Supplementary Figure 1 - see journal website).

The risk of PPM is strongly related to the size of implanted prosthetic valves $[7,8]$, with larger valves resulting in PPM less often than smaller valves. Differences in PPM prevalence were observed only in patients with medium-sized prostheses, where higher EOAi was observed in mini-AVR patients with PPM. Based on the mean value of EOAi in those patients, PPM may be categorised as moderate. A recent large meta-analysis found that severe PPM was associated with higher mortality [9]. It is possible that in mini-AVR surgeons were more often able to avoid implanting larger valves in favour of a medium-sized prosthesis, with acceptable postoperative haemodynamic parameters. In our study, postoperative transprosthetic gradients were slightly lower in mini-AVR. There were also no differences in transprosthetic gradients in mini-AVR patients with PPM compared to those without PPM, in contrast to con-AVR, where higher gradients were observed in the PPM group. Therefore, the higher prevalence of PPM in mini-AVR had no impact on postoperative haemodynamic parameters.

The effective orifice area can be measured in a variety of ways, and may change over time. EOAi can be calculated using the manufacturer's charts or echocardiographic data [5]. Due to the retrospective design of this analysis, the former method was used, which has been described elsewhere [4]. The EOAs reported by the manufacturer are based on in vitro models and their size may be overestimated in comparison with in vivo EOAs [5].

There are several surgical options to reduce the risk of PPM [10]. The first one involves aortic root enlargement procedures, which were not used in our study. Magruder et al. [5] show that aortic root enlargement procedures may also be successfully performed in mini-AVR patients to prevent PPM. The second option is the use of stentless bioprostheses, which present a better haemodynamic performance, but they are more challenging and increase the risk to the patient. New generation of stented bioprostheses is characterised by similar haemodynamic performance and an easier implanting technique [11]. There is no general recommendation for the preference of stentless bioprostheses. Most surgeons tend to implant stented valves in complicated cases and octogenarians because operative mortality and the stent durability are not worse than in stentless AVR [10]. In our analysis, no stentless bioprostheses were implanted.

At the time of publication, no standard objective patient selection criteria applied in our clinic for mini-AVR. The surgical approach was chosen by the individual surgeon. Therefore, 
it is possible that the higher frequency of PPM in mini-AVR is related to the surgeon learning the procedure.

Mini-AVR is associated with lower morbidity and mortality in high-risk patients (e.g. obese and elderly) [12]. On the other hand, studies on PPM showed decreased survival rates in patients $<70$ years of age and those with body mass index $<30 \mathrm{~kg} / \mathrm{m}^{2}$, but not in elderly or obese patients [13]. This may mean that there is an even stronger need to monitor mini-AVR procedures for PPM, because the benefits of this approach may be outweighed by the risk of worsened haemodynamics due to incorrect valve size.

This is a single-centre, non-randomised, retrospective study with a small patient sample size. The decision on the adopted method and the type and size of the implanted valve was a reflection of the individual surgeon's preference.

In conclusion, the incidence of PPM was found to be higher in patients undergoing mini-AVR compared to those undergoing conventional sternotomy.

\section{Conflict of interest: none declared}

\section{References}

1. Walther T, Rastan A, Falk V, et al. Patient prosthesis mismatch affects short- and long-term outcomes after aortic valve replacement. Eur J Cardiothorac Surg. 2006; 30(1): 15-19, doi: 10.1016/j. ejcts.2006.04.007, indexed in Pubmed: 16730449.

2. Mizia-Stec K, Mizia M, Sikora-Puz A, et al. Prosthesis-patient mismatch and left ventricle systolic strain in patients with severe degenerative aortic stenosis, who are undergoing surgical valve replacement. Kardiol Pol. 2016; 74(6): 537-546, doi: 10.5603/KP.a2015.0219, indexed in Pubmed: 26575313.

3. Koene BM, Soliman Hamad MA, Bouma W, et al. Impact of prosthesis-patient mismatch on early and late mortality after aortic valve replacement. J Cardiothorac Surg. 2013; 8: 96, doi: 10.1186/1749-8090-8-96, indexed in Pubmed: 23594366.

4. Pibarot P, Dumesnil JG. Prosthesis-patient mismatch: definition, clinical impact, and prevention. Heart. 2006; 92(8): 1022-1029, doi: 10.1136/hrt.2005.067363, indexed in Pubmed: 16251232.
5. Magruder JT, Grimm JC, Kilic A, et al. Mini-aortic valve replacements are not associated with an increased incidence of patient-prosthesis mismatch: a propensity-scored analysis. Gen Thorac Cardiovasc Surg. 2016; 64(3): 144-148, doi: 10.1007/s11748-015-0614-z, indexed in Pubmed: 26688505.

6. Bakir I, Casselman FP, Onan B, et al. Does a minimally invasive approach increase the incidence of patient-prosthesis mismatch in aortic valve replacement? J Heart Valve Dis. 2014; 23(2): 161-167, indexed in Pubmed: 25076545.

7. Hu J, Qian H, Li Yj, et al. Seventeen-millimeter St. Jude Medical Regent valve in patients with small aortic annulus: dose moderate prosthesis-patient mismatch matter? J Cardiothorac Surg. 2014; 9: 17, doi: 10.1186/1749-8090-9-17, indexed in Pubmed: 24438101.

8. Filip G, Bartuś K, Litwinowicz R, et al. Cardiac surgery early clinical outcomes of the surgical treatment of patients with aortic stenosis and small aortic annuli. Kardiochirurgia i Torakochirurgia Polska. 2013; 3: 199-203, doi: 10.5114/kitp.2013.38092.

9. Chen J, Lin Y, Kang Bo, et al. Indexed effective orifice area is a significant predictor of higher mid- and long-term mortality rates following aortic valve replacement in patients with prosthesis-patient mismatch. Eur J Cardiothorac Surg. 2014; 45(2): 234-240, doi: 10.1093/ejcts/ezt245, indexed in Pubmed: 23682010 .

10. Kobayashi J. Stentless aortic valve replacement: an update. Vasc Health Risk Manag. 2011; 7: 345-351, doi: 10.2147/VHRM. S11253, indexed in Pubmed: 21731886.

11. Bartus K, Litwinowicz R, Kusmierczyk M, et al. Primary safety and effectiveness feasibility study after surgical aortic valve replacement with a new generation bioprosthesis: one year ou comes. Kardiol Pol. 2018 [Epub ahead of print]; 76(3): 618-624, doi: 10.5603/KP.a2017.0262, indexed in Pubmed: 29297188.

12. Schmitto JD, Mohr FW, Cohn LH. Minimally invasive aortic valve replacement: how does this perform in high-risk patients? Curr Opin Cardiol. 2011; 26(2): 118-122, doi: 10.1097/HCO.0b013e328343983a, indexed in Pubmed: 21297462.

13. Mohty D, Dumesnil JG, Echahidi N, et al. Impact of prosthesis-patient mismatch on long-term survival after aortic valve replacement: influence of age, obesity, and left ventricular dysfunction. J Am Coll Cardiol. 2009; 53(1): 39-47, doi: 10.1016/j. jacc.2008.09.022, indexed in Pubmed: 19118723.

Cite this article as: Filip G, Litwinowicz R, Kapelak B, et al. Patient-prosthesis mismatch after minimally invasive aortic valve replacement. Kardiol Pol. 2018; 76(5): 908-910, doi: 10.5603/KP.2018.0096. 